\title{
Can't Humans Live According to the Sun, Not the Clock, Like Other Creatures on Earth?
}

\author{
Heon-Jeong Lee ${ }^{1,2}$ \\ 'Department of Psychiatry, Korea University College of Medicine, Seoul, Korea \\ ${ }^{2}$ Chronobiology Institute, Korea University, Seoul, Korea
}

Delayed sunrise time in winter makes it difficult to wake up and start the day at the usual wake-up time. Is it really normal to get up early and start the day long before sunrise during winter? All living things on Earth, except mankind, live according to sunrise and sunset. Diurnal animals usually initiate their activities at sunrise, and nocturnal animals generally start after sunset. Would not this pattern of humans, as a diurnal animal, that goes against the sunrise time, have an adverse effect on human life and health?

Before humans developed electricity and artificial lighting, they generally began their daily routine when the sun rose. However, the availability of artificial lighting has created an environment wherein activities are possible even without sunlight. As a result, humans now live their daily lives according to the set time indicated by the clock, and not sunlight. In other words, even though there are variations in sunrise time between seasons, people live according to the standard time displaced by the clock. For example, humans still wake up at 6:00 AM when it is dark in the winter and get ready to go to school or work. Though this generally reflects the sunrise time, it does not take into account the seasonal changes associated with sunrise. For instance, the sunrise time in Seoul, South Korea, in 2021 is 5:11 AM and 7:43 AM in the summer and winter solstice, respectively, which is a difference of $2 \mathrm{~h}$ and $32 \mathrm{~min}$.

Since the sun rises earlier in summer and later in winter, people consider it natural that the sunlight shines in the bedroom much

Received: November 27, 2021 Revised: December 8, 2021

Accepted: December 8, 2021

Corresponding author: Heon-Jeong Lee, MD, PhD, Department of Psychiatry, Anam Hospital, Korea University College of Medicine, 73 Goryeodae-ro, Seongbuk-gu, Seoul 02841, Korea.

Tel: 82-2-920-5815, E-mail: leehjeong@korea.ac.kr

(a) This is an Open Access article distributed under the terms of the Creative Commons Attribution Non-Commercial License (https://creativecommons.org/ licenses/by-nc/4.0) which permits unrestricted non-commercial use, distribution, and reproduction in any medium, provided the original work is properly cited. earlier before waking up in summers and getting up in the dark during winters to begin their day. However, this is not as natural as humans perceive; this is an artificial phenomenon that only humans experience among living things on Earth. Living life according to artificial clocks can lead to deviations between the biological internal circadian rhythms and the external rhythms, which can cause various health problems such as sleep, mood, and metabolic disorders.

Then, is there a solution? Can daylight saving time (DST) be an alternative? DST is in the process of advancing an hour from spring to autumn and then delaying it again. This does seem like a solution, but advancing an hour in March and then delaying it in November can be too abrupt, causing problems in circadian rhythm adaptation. Several studies have reported various problems that arise due to the implementation of DST [1,2]. This is because the natural adaptation process of human biological clocks was not well considered when implementing DTS; the primary reason for this implementation was economic efficiency by using more sunlight during the day.

Recent advances in technology will enable the development of a system that can slowly advance and delay time according to the seasonal variations in sunrise time. Because the sun rises earlier in summer, the system should work towards gradually advancing the time from winter to summer. On the other hand, as the sun rises later in winter, the system should steadily delay the time from summer to winter.

A challenge in implementing this gradual advancing/delaying system will be sharing the standard time across the world, such as determining the time of events held in other parts of the Earth, like the departure and arrival times of airplanes and trains. However, it is certainly not difficult with the current technology to convert local times to international standard times (Coordinated Universal Time). Thus, humans may live with their biological rhythms synchronized to the local sunrise time and still not miss 
out on important meetings in other countries with the help of technology. Although more discussions should precede toward finding a way to gradually advance and delay time throughout the year, the current level of technology is advanced enough to provide a sufficient solution.

To live a more stable and healthy life, it is time to discuss finding a way to live according to the sun so that humans, like other creatures on Earth, can maintain their natural biological rhythms in seasonal changes.

Acknowledgments

This study was supported by the Korea Health 21 R\&D Project funded by the National Research Foundation of Korea (2017M3 A9F1031220).
Conflicts of Interest

The author has no potential conflicts of interest to disclose.

ORCID iD

Heon-Jeong Lee (i)

https://orcid.org/0000-0002-9560-2383

\section{REFERENCES}

1. Fritz J, VoPham T, Wright KP Jr, Vetter C. A chronobiological evaluation of the acute effects of daylight saving time on traffic accident risk. Curr Biol 2020;30:729-735.

2. Manfredini R, Fabbian F, De Giorgi A, Zucchi B, Cappadona R, Signani F, et al. Daylight saving time and myocardial infarction: should we be worried? A review of the evidence. Eur Rev Med Pharmacol Sci 2018;22:750-755. 